\title{
Antibody response after hepatitis B immunisation in a group of health care workers
}

\author{
A Cockcroft, P Soper, C Insall, Y Kennard, S Chapman, C Gooch, P Griffiths
}

\begin{abstract}
Hepatitis $B$ immunisation has been offered to staff of Hampstead Health Authority since 1982 and is now offered to all staff with clinical contact. Three doses of $20 \mu \mathrm{g}$ of vaccine are given at zero, one, and six months and the antibody response is measured three months later. Results were analysed to seek for associations with the antibody response. At the time of analysis, 2739 people had started vaccination and 1067 had completed the course and had a measurement of antibody response. Vaccine injections were initially into the buttock and later into the arm; measurement of antibody levels was initially by radioimmunoassay (RIA) and later by enzyme immunoassay (EIA). A positive antibody response was defined as a positive/negative ratio of $>10$ for RIA or a level of $>10 \mathrm{mIU} / \mathrm{ml}$ for EIA. Associations between antibody response and other variables were tested by $\chi^{2}$ and a multiple logistic regression analysis was undertaken to examine the effects of variables in combination. The overall antibody response rate was 95\%. Men and women did not respond differently but there were significantly more positive responses with the EIA testing method and a tendency for more positive responses with arm injections. The responders were significantly younger than the non-responders and had significantly lower values of body mass index $\left(w t / \mathbf{h t}^{2}\right)$. There were significant interactions between sex/injection site and body mass index/injection site, such that antibody response rate fell sharply with higher values of body mass index, but only for buttock injections, and women had a slightly better response than men for arm injections but a slightly worse response for buttock injections. The group least likely to respond were women
\end{abstract}

Occupational Health Unit and Departments of Clinical Epidemiology and General Practice and Virology, Royal Free Hospital and School of Medicine, London NW3 2QG

A Cockcroft, P Soper, C Insall, Y Kennard, S Chapman, C Gooch, P Griffiths with high values of body mass index given buttock injections. This may be because the vaccine is injected into fat rather than muscle in these individuals and is only poorly presented to the immune system, so eliciting little immune response. With injections into the arm and using the EIA method of antibody measurement, the response rate in this population is around $99 \%$. Thus routine antibody testing after immunisation is probably unnecessary.

Hepatitis B immunisation, using plasma derived vaccine, is a safe and effective means of protecting against hepatitis $B$ infection in groups of individuals at risk. ${ }^{2}$ In 1987 recombinant hepatitis B vaccine was licensed in the United Kingdom. Immunisation with either preparation is recommended for health care workers, especially those at risk of contamination with blood or other body fluids in the course of their work. ${ }^{3}$ The seroconversion rate after a course of three injections of vaccine is generally high. ${ }^{45}$ The proportion of non-responders increases with age ${ }^{45}$ and has been reported to be higher when the vaccine is given into the buttock than when it is given into the upper arm. ${ }^{6}$ Some non-responders may respond to a fourth dose of vaccine $e^{57}$ but there is apparently a genetically determined group of persistent non-responders. ${ }^{8}$

A programme of hepatitis $B$ immunisation for staff began in Hampstead Health Authority soon after the plasma derived vaccine was licensed in the United Kingdom. The antibody response to the vaccine was measured as part of this programme and this has allowed an analysis of the variables related to the response rate to be undertaken. This paper describes the immunisation programme and presents the results of the analysis of the antibody response.

\section{Subjects and methods}

IMMUNISATION PROGRAMME

The programme began in late 1982 . Initially staff identified as being at particular occupational risk of hepatitis B were included, such as those working in the liver, renal, and haemophilia units. The programme gradually expanded so that now the vaccine is being offered to all staff in clinical contact, including trained and student nurses, medical 
students and doctors, laboratory staff, some technical staff, porters, central sterile supply department workers, some laundry workers, and some domestic staff. At the time of the analysis reported here, 2739 staff members had begun an immunisation course in the district.

The immunisation programme is undertaken by the district occupational health unit. Since 1987 the programme has been computerised, using a microcomputer in the occupational health unit and dBase III + software (Ashton-Tate). Information was extracted from this database for analysis.

The immunisation course consists of three doses, each of $20 \mu \mathrm{g}$, of vaccine at zero, one, and six months. Initially, injections were given into the buttock (aiming for the gluteal muscles) but this practice was changed after reports of lower response rates with injections at this site. ${ }^{6}$ Thereafter, the vaccine was injected into the upper arm (aiming for the deltoid muscle). Thus some people had mixed site courses of vaccine. The vaccine used was a plasma derived type for most of the period of the programme analysed here but a recombinant type was used in a few subjects near the end of the period and has now become the routine type used.

Anti-HBs antibody was measured before immunisation in the early days of the programme but this practice was discontinued because so few people $(<1 \%)$ in this population had pre-existing antibodies. In all individuals antibodies were measured three months after the third dose of vaccine. The initial method used to estimate anti-HBs levels was a commercially available radioimmunoassay (RIA) (Abbott Laboratories). For organisational reasons, the department measuring the antibody levels changed in the summer of 1987 and the new department used an enzyme immunoassay (EIA) method for estimating anti-HBs (Behring). All the subjects who had antibodies tested by the EIA method had received injections into the arm.

\section{INFORMATION FOR THE ANALYSIS}

Subjects included in the analysis were those who had received three doses of vaccine (at zero, one, and six months) and who had had a blood sample taken for estimation of anti-HBs about 12 weeks (range 4-26 weeks) after the third injection. As at August 1988 2739 subjects had received at least one vaccine dose, 2128 had received three doses, and 1067 had received three doses and had a measurement of antibody response.

Information recorded for each subject included age, sex, height, weight, site of injections, postimmunisation anti-HBs levels, and method of antibody estimation for each recorded level. The Quetelet index of body mass (weight/height ${ }^{2}$ ) was calculated for each subject and used in the analysis. ${ }^{9}$
For antibodies measured by RIA, a positive-negative ratio of $>10$ was considered as a positive response and of $<10$ as a negative response. For EIA measurements antibody levels of $>10 \mathrm{mIU} / \mathrm{ml}$ were considered a positive response and those $<10 \mathrm{mIU} /$ $\mathrm{ml}$ were considered a negative response.

\section{ANALYSIS}

Univariate analysis was performed to determine differences in age and Quetelet index for positive and negative antibody responders and the effect on antibody response, positive or negative, of the variables sex, site of vaccine injection, and method of measuring anti-HBs. The significance of differences was tested using the Mann-Whitney test or $\chi^{2}$ test as appropriate.

A multiple logistic regression analysis (using the GLIM statistical package) was then performed to examine the relation between the proportion of positive antibody responses and the same variables in combination. ${ }^{10}$ Logistic regression models were fitted separately for all the data, for antibody results by RIA only, and for antibody results by EIA only. In each case the model for the contributions of the different variables that best explained the antibody results was sought. The analysis was confined to subjects with complete data for all the variables and who had received injections at only one site, whether buttock or arm.

\section{Results}

In the 1067 subjects included in the analysis the overall response rate (anti-HBs $>10$ positivenegative ratio or $>10 \mathrm{mIU} / \mathrm{ml}$ ) was approximately $95 \%$. The response rate was significantly higher when anti-HBs was estimated by the EIA rather than the RIA method (table 1). The response rate did not differ between men and women with either method of testing (table 2). The response rate tended to be higher in those who had received injections in the arm rather than the buttock (excluding those who had had mixed site injections), although the difference was not significant at the $10 \%$ level (table 3 ). For both testing methods, responders were significantly younger than non-responders and had significantly lower values of Quetelet index (table 4). The number of subjects who received the recombin-

Table 1 Effect of testing method (RIA or EIA) on measured antibody response about 12 weeks after immunisation

\begin{tabular}{lcc}
\hline & RIA method & EIA method \\
\hline Total no tested & 398 & 669 \\
No of responders & 353 & 661 \\
No of non-responders $(\%)$ & $45(11 \cdot 3)$ & $8(1 \cdot 2)$ \\
\hline
\end{tabular}

Responders and non-responders are as defined in the text. The difference between the response rate for the two testing methods is significant $\left(\chi^{2} 54.04, \mathrm{p}<0.00001\right)$. 
Table 2 Effect of sex on the antibody response after immunisation

\begin{tabular}{lcc}
\hline & Men & Women \\
\hline & RIA testing method \\
Total no tested & 145 & 253 \\
No of responders & 128 & 225 \\
No of non-responders (" $\left.{ }^{\circ}\right)$ & $17(11 \cdot 7)$ & $28(11 \cdot 1)$ \\
& EIA testing method \\
Total no tested & 158 & 508 \\
No of responders & 155 & 503 \\
No of non-responders $\left({ }^{\prime}{ }_{0}\right)$ & $3(1 \cdot 9)$ & $5(1 \cdot 0)$ \\
\hline
\end{tabular}

Responders and non-responders as defined in the text. The response rate does not significantly differ between the sexes for either testing method.

ant vaccine (all of whom had antibody levels measured by EIA) was too small for separate analysis but there was no suggestion of a response rate differing between the two types of vaccine.

The logistic regression analysis was performed on 891 subjects with complete data for all variables: 250 with antibodies tested by RIA and 641 with antibodies tested by EIA. The variation in proportion of positive antibody responses was significantly reduced by taking into account the combination of the one way effects of the measured variables, except when only considering antibody results by EIA where there was only one site of injection (the arm). For all the data, and for those including only antibodies measured by RIA, further significant reductions in variation were produced by adding the two way interactions of sex injection site and Quetelet index injection site. There was a significant effect of age and antibody testing method which was uniform for all levels of sex, injection site, and Quetelet index. But the influence of injection site on the response differed for the two sexes and for different levels of Quetelet index.

Figure 1 shows the effect of age on the proportion of positive responders for the RIA testing method only, predicted from the best fitting model. Figure 2 illustrates the predicted effects of the interactions between sex, injection site, and Quetelet index on the proportion of positive responders at the median age of 27. For arm injections, the response is slightly higher for women than men, whereas for buttock injections, men have a higher rate of response. The rate of response declines gradually with increasing

Table 3 Effect of injection site on the antibody response after immunisation, using the $R I A$ testing method

\begin{tabular}{|c|c|c|}
\hline & Buttock & Arm \\
\hline Total no tested & 59 & 217 \\
\hline No of responders & 51 & 199 \\
\hline No of non-responders $\left({ }^{\prime}{ }_{1}\right)$ & $8(13.6)$ & $18(8 \cdot 3)$ \\
\hline
\end{tabular}

Responders and non-responders as defined in the text. Subjects who had mixed site injection courses are excluded. The difference in response rate between the injection sites is not significant at the $10^{\prime \prime}$." level $\left(x^{2} 1.51\right)$.
Table 4 Effect of age and Quetelet index on the antibody response after immunisation for the RIA and EIA testing methods

\begin{tabular}{|c|c|c|c|c|}
\hline & \multicolumn{2}{|c|}{ Age $y$} & \multicolumn{2}{|c|}{ Quetelet index } \\
\hline & No & Median & No & Median \\
\hline \multicolumn{5}{|c|}{$R I A$ testing method } \\
\hline Responders & 339 & $26 \cdot 0$ & 319 & $22 \cdot 2$ \\
\hline Non-responders & 44 & 33.5 & 38 & 23.6 \\
\hline $\mathrm{p}$ Value of difference & \multicolumn{2}{|c|}{$<0 \cdot 0001$} & \multicolumn{2}{|c|}{$<0 \cdot 005$} \\
\hline \multicolumn{5}{|c|}{ EIA testing method } \\
\hline Responders & 655 & $23 \cdot 0$ & 645 & $22 \cdot 2$ \\
\hline Non-responders & \multirow{2}{*}{\multicolumn{2}{|c|}{$\begin{array}{ll}8 & 26.0 \\
<0.05 & \end{array}$}} & 8 & $24 \cdot 7$ \\
\hline $\mathrm{p}$ Value of difference & & & \multicolumn{2}{|c|}{$<0.005$} \\
\hline
\end{tabular}

Age and Quetelet index were not available for some subjects Responders and non-responders as defined in the text. The median age of the subjects tested by the RIA method $27 \mathrm{y})$ was significantly greater than that of those tested by the EIA method (23 y) p $<0.00005$

Quetelet index for arm injections but falls rapidly with values of the index above 26 for buttock injections. The lowest predicted proportion of positive responses is in women with buttock injections and high values of Quetelet index.

\section{Discussion}

Our results confirm and extend those of previous studies of hepatitis B immunisation among health care workers. ${ }^{45}$ Response rates after three injections (anti-HBs $>10 \mathrm{mIU} \mathrm{ml}$ ) have been reported to be around $92^{\prime \prime} "{ }^{5}$ Overall, we found a response rate of around 95" ", with an important effect of method of

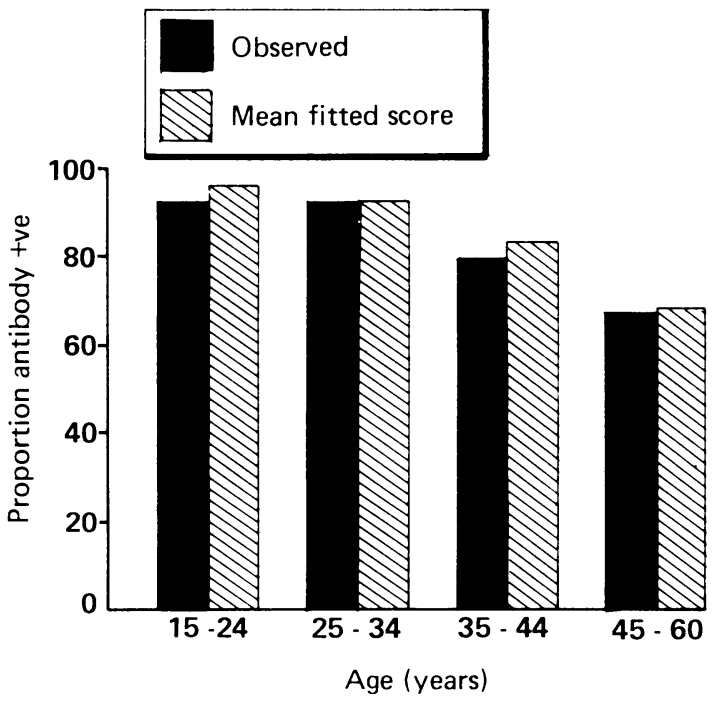

Figure 1 Average of predicted probabilities and actual proportions of positive antibody responses for different age groups. Predicted probabilities are derived from best fitting logistic regression equation, taking into account effects of other variables. Only antibody responses tested by $R I A$ method are included. A positive response is as defined in text. 


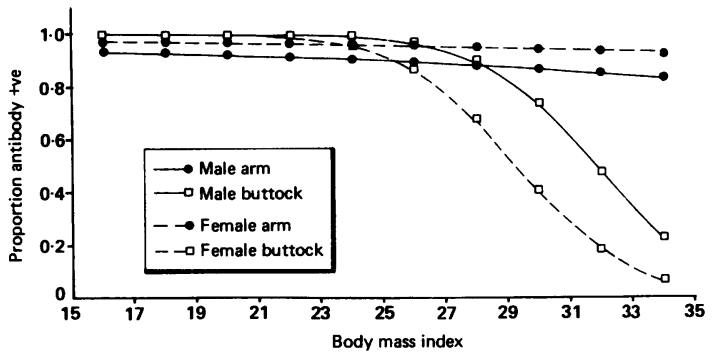

Figure 2 Predicted proportion of positive antibody responses at different values of Quetelet Index for different combinations of sex and injection site at median age of 27, predicted from best fitting logistic regression equation. Only antibody responses tested by $R I A$ method are included. Positive response is as defined in text.

testing. Since not only the method but also the department undertaking the testing changed, we cannot be sure whether some aspect of the test itself is responsible for the much lower non-response rate with the EIA method. Although there was a proportion of subjects vaccinated into the buttocks and a significantly higher median age with the RIA testing method, this did not explain the difference between testing methods, since method of testing was related to the antibody response for arm injections and for all levels of age. The level taken as the "cut-off" point to determine a positive or negative response by the two methods may not be equivalent. But this is unlikely to be a full explanation as we now know that most of the levels measured by the EIA technique were much higher than the $10 \mathrm{mIU} / \mathrm{ml}$ cut-off point (P Griffiths, personal communication).

The effect of age on antibody response in our data agrees with other studies. ${ }^{45}$ It was independent of the effects of other variables. The effect was pronounced only in those older than 35 but was nevertheless a strong feature of the data.

Antibody response has been reported to be better in women, ${ }^{4}$ though not all investigators agree. ${ }^{5} \mathrm{We}$ found no overall effect of sex on antibody response but a significant interaction with injection site, such that women were less likely to respond than men if the vaccine was given into the buttock with the reverse if the vaccine was given into the arm. This interaction may help to explain the discrepancy between the results of other investigators. Despite reports of a poorer response to injections in the buttock, ${ }^{6}$ recent authors have reported series of immunisations into the buttock with good response rates. ${ }^{57}$ We were able to look at the effect of injection sites within our series and have found that the effect of injection site is mainly in subjects with a high Quetelet index (fig 2). This interaction has not previously been taken into account. The probable explanation for the interaction is that in obese people buttock injections are intra-adipose rather than intramuscular. The different distribution of adipose tissue in men and women may explain why this effect seems to be especially evident in women (see fig 2). Presumably the vaccine is only poorly presented to the immune system from the adipose tissue and so elicits little immune response.

Using our present antibody testing method, and injecting vaccine into the arm, our non-response rate is in the order of $1 \%$. This suggests that routine testing of postimmunisation antibody levels is probably unnecessary.

1 Szmuness W, Stevens CE, Zang EA, Kellner A. A controlled trial of the efficacy of the hepatitis B vaccine (Hepatavax B): a final report. Hepatology 1981;5:377-85.

2 Francis DP, Hadler SC, Thompson SE, et al. The prevention of hepatitis B with vaccine: report of the Centers for Disease Control multi-center efficacy trial among homosexual men. Ann Intern Med 1982;97:362-6.

3 Department of Health and Social Security. Immunisation against infectious disease. London: HMSO, 1988.

4 Deinhardt F. Aspects of vaccination against hepatitis B: passiveactive immunisation schedules and vaccination responses in different age groups. Scand J Infect Dis 1982;38:17-23S.

5 Fagan EA, Tolley P, Smith HM, et al. Hepatitis B vaccine: immunogenicity and follow-up including two year booster doses in high risk health care personnel in a London teaching hospital. J Med Virol 1987;21:49-56.

6 Centre for Disease Control. Suboptimal response to hepatitis B vaccine given by injection into the buttock. $M M W R$ 1985;34:105-8, 113.

7 Campbell AD, Hambling MH, Pinnington PA, Lees E. Efficacy of hepatitis B vaccine. $J$ Soc Occup Med 1988;38:46-7.

8 Nowicki MJ, Tong MJ, Bohman RE. Alterations in the immune response of nonresponders to the hepatitis B vaccine. J Infect Dis 1985;152:1245-8.

9 Rosenbaum S. 100 years of heights and weights. Journal of the Royal Statistical Society 1988;151:276-309.

10 Dobson AJ. An introduction to statistical modelling. London: Chapman and Hall, 1983:74-87.

Accepted 3 July 1989 